\title{
Pulmonary aspiration in preschool children with cystic fibrosis
}

\author{
D. Clarke ${ }^{1,2}$, I. Gorman ${ }^{1,2^{*}}$, F. Ringholz ${ }^{1,2}$, M. McDermott ${ }^{2}$, DW. Cox ${ }^{1,2}$, P. Greally ${ }^{3}$, B. Linnane ${ }^{1,5,6}$ and P. Mc Nally $y^{1,2,4}$
}

\begin{abstract}
Pulmonary aspiration of gastric refluxate (PAGR) has been demonstrated in association with pulmonary inflammation in school aged children with Cystic Fibrosis (CF). We sought to determine if similar findings were present in preschool children. Pepsin was measured in Broncho-alveolar lavage (BAL) fluid collected from clinically stable preschool children with CF and controls. Elevated pepsin levels were found in a subgroup of children with CF, but this was not found to be associated with pulmonary infection, pulmonary inflammation or respiratory or gastrointestinal symptoms.
\end{abstract}

\section{Introduction}

Gastro-oesophageal reflux (GOR) is the involuntary passage of gastric content into the oesophagus. Gastro-oesophageal reflux disease (GORD) is present when GOR causes adverse consequences such as pain, failure to thrive or pulmonary aspiration. The reported prevalence of GORD in CF is between 35 and $81 \%$ and has been associated with reduced lung function and increased cough in older patients $[1,2]$. We have previously demonstrated that PAGR occurs in a subgroup of school-aged children with CF and is associated with more pronounced airway inflammation [3].

There has been much focus recently on the detection of evolving lung disease in preschool children with CF, where traditional outcome measures are poor and children can be largely asymptomatic despite the development of irreversible structural lung disease $[4,5]$. The focus of pulmonary management in CF is increasingly moving towards aggressive prevention of disease, where possible, rather than solely targeting clinically evident complications. Pulmonary aspiration can cause significant lung disease [6] but is difficult to diagnose clinically [7]. We sought to discover if pulmonary aspiration was occurring in a group of clinically stable preschool children with $\mathrm{CF}$ and, if present, was associated with greater airway inflammation.

\footnotetext{
* Correspondence: igorman@tcd.ie

${ }^{1}$ National Children's Research Centre, Crumlin, Dublin 12, Ireland

${ }^{2}$ Our Lady's Children's Hospital, Crumlin, Dublin 12, Ireland

Full list of author information is available at the end of the article
}

\section{Methods}

Clinically stable preschool children with CF undergoing routine surveillance bronchoscopy were recruited through the Study of Host Immunity and Early Lung Disease in Cystic Fibrosis (SHIELD CF). Clinical data were prospectively collected on all children at CF clinic with a standardised clinical information sheet. Non-CF controls were children without CF undergoing bronchoscopy for clinical reasons. Informed parental consent was obtained and the study had ethical approval from the institutional review board of Our Lady's Children's Hospital, Crumlin, Dublin.

Bronchoscopy was performed under general anaesthesia via laryngeal mask airway. Bronchoalveolar lavage (BAL) samples were obtained by instilling two sequential aliquots of $1 \mathrm{ml} / \mathrm{kg}$ of sterile normal saline per lobe, into the right middle lobe and lingula (or other affected lobe). Retrieved samples were then pooled. Differential cell counts were performed microscopically after staining with Haematoxylin and Eosin. Pepsin concentrations were measured in control and CF BAL using a commercial human pepsin ELISA (USCN Life Science Inc. China). Interleukin 8 (IL-8) was measured in CF BAL by ELISA as previously described [3]. Neutrophil Elastase (NE) activity of CF BAL samples was quantified using the substrate $\mathrm{N}$ - (Methoxysuccinyl)-Ala-Ala-Pro-Val p-nitroanilide as previously described [8]. Differences in normally distributed data were analysed by Students t-test, chi squared or Pearson, and non-normally distributed data with Mann Whitney and Spearman. Significance is denoted as a $p$ value $<0.05$. 
Table 1 Baseline demographics for the study populations

\begin{tabular}{llll}
\hline & CF $(n=77)$ & Controls $(n=12)$ & $p$ value \\
\hline Age, mean in years (range) & $3.75(0.86-6.4)$ & $3.6(1.5-7.8)$ & 0.747 \\
Gender & $26 \mathrm{~F} / 51 \mathrm{M}$ & $3 \mathrm{~F} / 9 \mathrm{M}$ & 0.547 \\
BMl, mean (range) & $16.6(15-20.6)$ & $17.6(16.1-19.7)$ & 0.156 \\
Genotype & & & \\
F508del Homozygotes & 50 & $\mathrm{~N} / \mathrm{A}$ & $\mathrm{N} / \mathrm{A}$ \\
F508del Heterozygotes & 17 & $\mathrm{~N} / \mathrm{A}$ & $\mathrm{N} / \mathrm{A}$ \\
Other & 10 & $\mathrm{~N} / \mathrm{A}$ & $\mathrm{N} / \mathrm{A}$ \\
\hline
\end{tabular}

\section{Results}

Eighty nine children were included in the study, 77 with CF [mean age 3.7 years (range 0.86-6.4)] and 12 Control [3.6 years (1.5-7.8)] (Table 1). No patients had more than one BAL. BAL pepsin levels were not significantly different between the groups $(385 \pm 68.7 \mathrm{pg} / \mathrm{ml} \mathrm{v.} 198 \pm$ $54.2 \mathrm{pg} / \mathrm{ml}$ [mean \pm SEM]) (Fig. 1a). A subgroup of children with CF (18/77 [23\%]) had particularly high levels (high pepsin, $>2$ SD above control mean [>573 pg/ml normally distributed data]) compared to all others with CF (low pepsin). There was no difference in levels of IL-8, BAL neutrophil counts, proportion with free NE in BAL or NE levels (where detected) between the high and low pepsin groups (Fig. 1b, d, e). No associations were found between BAL levels of pepsin and either IL-8 or NE (where detected) (Fig. 1c, f). Recognised CF pathogens ( $S$. aureus, $H$. influenza, $P$. aeruginosa, S. maltophilia) were found in BAL from 41 of 77(53\%) children with CF. There was no statistically significant difference in the presence of these organisms, either individually or collectively between the high and low pepsin groups. There was a low prevalence of $P$. aeruginosa (3.9\%) and MRSA (1.3\%) overall within the CF group. There was no significant difference in any of the gastrointestinal (3/18 and 5/59 with GOR symptoms $[p=0.136])$ or respiratory symptoms related to pulmonary aspiration or GOR between children with CF with high and low pepsin levels.

\section{Discussion}

We demonstrate in this study that almost 1 in 4 preschool children with CF have elevated levels of BAL pepsin, suggesting sub-clinical PAGR. In contrast to the findings of our previous study in older children with CF [3], in this study there is no evidence that the presence of pepsin in BAL was associated with pulmonary inflammation. We have little understanding of the natural history of PAGR in children with CF, particularly as it relates to frequency of occurrence and variability over time. PAGR may need to be present for a number of years before it is associated with recognisable pulmonary inflammation. This will require further study.

We specifically prospectively collected clinical data on respiratory and gastrointestinal symptoms (heartburn, vomiting, post-tussive vomiting, coughing pattern) to

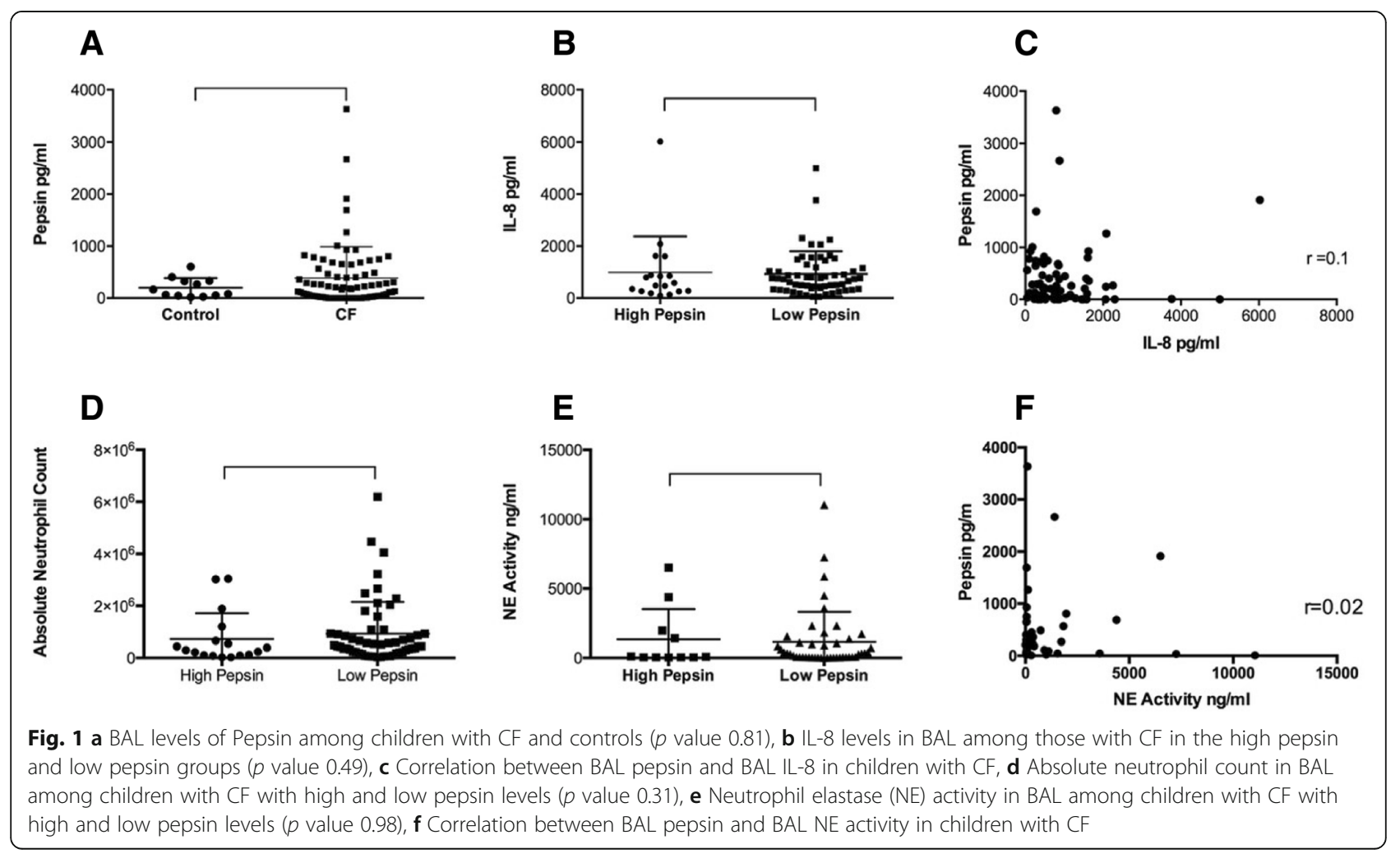


attempt to determine if there was a recognisable clinical phenotype associated with PAGR. We found no difference in symptoms in those with high pepsin levels compared to the rest of the CF group. The diagnosis and treatment of PAGR is a challenging area. There is likely a significant overlap between the symptoms of pulmonary aspiration and typical lung disease in $\mathrm{CF}$, and while it is possible to measure GOR, and to radiologically assess pulmonary aspiration during swallowing, we lack a specific and sensitive commercially available diagnostic test for the aspiration of refluxate, the key biological event of concern. Because we have no available treatment to prevent aspiration of material that refluxes into the oesophagus or pharynx, treatment for PAGR involves prevention of GOR, which may ultimately entail fundoplication, not an insignificant undertaking. Proton pump inhibitors, commonly used in CF, may well worsen the situation $[9,10]$.

While it is positive that neither changes in pulmonary pathogens or pulmonary inflammation were found in those with PAGR, we have nonetheless discovered a potentially significant biological abnormality that occurs in a subset of patients. Unfortunately, a perfect biomarker for pulmonary aspiration does not exist. Longitudinal BAL pepsin measurements, perhaps in combination with other previously published PAGR biomarkers such as bile acids [2], with robust medium term outcome measures, will be required to determine if early PAGR is associated with subsequent clinically relevant adverse outcomes, an approach we plan with this cohort.

\section{Abbreviations \\ BAL: Broncho-alveolar lavage; CF: Cystic fibrosis; ELISA: Enzyme linked immunosorbent assay; GOR: Gastro-oesophageal reflux; GORD: Gastro- oesophageal reflux disease; IL-8: Interleukin 8; NE: Neutrophil elastase; PAGR: Pulmonary aspiration of gastric refluxate; SHIELD CF: Study of host immunity and early lung disease in cystic fibrosis}

\section{Acknowledgements}

Not applicable.

\section{Funding}

This work was funded by the National Children's Research Centre in Ireland. The sponsor had no role in study design, data interpretation or analysis.

\section{Availability of data and materials}

The datasets used and/or analysed during the current study are available from the corresponding author on reasonable request.

\section{Authors' contributions}

DC was involved with study design, patient recruitment, sample preparation, processing and analysis, and manuscript preparation. IG was involved with sample preparation and processing, and manuscript preparation. FR was involved with sample preparation, processing and analysis. MMcD was involved with histology sample preparation and analysis. DC, PG and BL were involved with patient recruitment, sample collecting, processing and analysis. PMCN was involved with study design, patient recruitment, sample collecting, analysis, manuscript preparation. All authors provided manuscript edits, and read and approved the final manuscript.

\section{Ethics approval and consent to participate}

Ethics approval was obtained for this study from the Our Lady's

Children's Hospital Ethics committee as part of the SHIELD CF research programme (The Study of Host Immunity and Early Lung Disease in CF).

\section{Consent for publication}

Not applicable

\section{Competing interests}

The authors declare that they have no competing interests.

\section{Publisher's Note}

Springer Nature remains neutral with regard to jurisdictional claims in published maps and institutional affiliations.

\section{Author details}

${ }^{1}$ National Children's Research Centre, Crumlin, Dublin 12, Ireland. ${ }^{2}$ Our Lady's Children's Hospital, Crumlin, Dublin 12, Ireland. ${ }^{3}$ National Children's Hospital, Tallaght, Dublin, Ireland. ${ }^{4}$ Department of Paediatrics, Royal College of Surgeons in Ireland, Dublin, Ireland. ${ }^{5}$ University Hospital Limerick, Limerick, Ireland. ${ }^{6}$ Graduate Entry Medical School and Centre for Interventions in Infection, Inflammation \& Immunity (4i), University of Limerick, Limerick, Ireland.

Received: 3 June 2018 Accepted: 23 November 2018

Published online: 17 December 2018

\section{References}

1. Blondeau K, Pauwels A, Dupont L, et al. Characteristics of gastroesophageal reflux and potential risk of gastric content aspiration in children with cystic fibrosis. J Pediatr Gastroenterol Nutr. 50(2):161-6.

2. Pauwels A, Decraene A, Blondeau K, et al. Bile acids in sputum and increased airway inflammation in patients with cystic fibrosis. Chest. 141(6):1568-74.

3. McNally P, Ervine E, Shields MD, et al. High concentrations of pepsin in bronchoalveolar lavage fluid from children with cystic fibrosis are associated with high interleukin-8 concentrations. Thorax. 2011;66(2):140-3.

4. Sly PD, Gangell $\mathrm{CL}$, Chen $\mathrm{L}$, et al. Risk factors for bronchiectasis in children with cystic fibrosis. N Engl J Med. 2013;368(21):1963-70.

5. Stick $\mathrm{S}$, Tiddens $\mathrm{H}$, Aurora $\mathrm{P}$, et al. Early intervention studies in infants and preschool children with cystic fibrosis: are we ready? Eur Respir J. 2013;42(2):527-38.

6. Boesch RP, Daines C, Willging JP, et al. Advances in the diagnosis and management of chronic pulmonary aspiration in children. Eur Respir $J$. 2006;28(4):847-61.

7. Farrell S, McMaster C, Gibson D, et al. Pepsin in bronchoalveolar lavage fluid: a specific and sensitive method of diagnosing gastro-oesophageal refluxrelated pulmonary aspiration. J Pediatr Surg. 2006;41(2):289-93.

8. Weldon S, McNally P, McElvaney NG, et al. Decreased levels of secretory leucoprotease inhibitor in the pseudomonas-infected cystic fibrosis lung are due to neutrophil elastase degradation. J Immunol. 2009;183(12): 8148-56.

9. Pauwels $A$, Verleden $S$, Farre $R$, et al. The effect of gastric juice on interleukin-8 production by cystic fibrosis primary bronchial epithelial cells. J Cyst Fibros. 2013;12(6):700-5.

10. Dimango $E$, Walker $P$, Keating $C$, et al. Effect of esomeprazole versus placebo on pulmonary exacerbations in cystic fibrosis. BMC Pulm Med. 2014;14:21. 